SCIPP-2008/06

\title{
Symmetric Points in the Landscape as Cosmological Attractors
}

\author{
Michael Dine, Guido Festuccia and Alexander Morisse \\ Santa Cruz Institute for Particle Physics and \\ Department of Physics, University of California, Santa Cruz CA 95064
}

\begin{abstract}
In the landscape, if there is to be any prospect of scientific prediction, it is crucial that there be states which are distinguished in some way. The obvious candidates are states which exhibit symmetries. Here we focus on states which exhibit discrete symmetries. Such states are rare, but one can speculate that they are cosmological attractors. We investigate the problem in model landscapes and cosmologies which capture some of the features of candidate flux landscapes. In non-supersymmetric theories we find no evidence that such states might be cosmologically favored. In supersymmetric theories, simple arguments suggest that states which exhibit $R$ symmetries might be. Our considerations lead us to raise questions about some popular models of eternal inflation.
\end{abstract}




\section{Introduction: Distinguished States in the Landscape}

For almost 30 years, inspired by string theory, particle theorists have imagined that there is a sensible program of attempting to infer the laws of nature from some grand set of principles from the top down. The emergence of the string landscape, however, has thrown this paradigm into question. If there is any truth to the idea of a cosmic landscape[1, 2, 3, 4, 5], there is no realistic program in which one finds the string ground state which describes the world around us. At best, one can hope to do statistics, looking for strong correlations between known facts and laws of nature which can be studied in future experiments.

At present, even an enthusiast must acknowledge that the landscape is more of a vision than a well-defined theoretical structure. At best, we have explored only a small subset of the possible set of states $[6,7]$; most of these are supersymmetric or approximately so. The most interesting states, with broken supersymmetry and small cosmological constant, can hardly be said to have been understood in a systematic way. In the original KKLT construction, for example, while there are small numbers, there is not a formal, small parameter in which one can develop a systematic approximation scheme. On the other hand, the KKLT analysis seems plausible, and the statistics which arise from this and similar constructions seem robust. For example, the statistics discussed by Douglas and Denef[8, 9] emerge from simple assumptions about the low energy degrees of freedom, as well as assumptions of uniform distributions of lagrangian parameters with respect to some natural measure[10]. So it is perhaps not unreasonable to think that these model landscapes may provide reliable insights into the larger structure.

It is not at all clear whether the (nearly) supersymmetric states are representative; serious arguments have been advanced that they are not $[9,11]$. So, even accepting the validity of the landscape framework, it is unlikely that we will soon be able to reliably enumerate all possible classes of landscape vacua. Thus if there is to be any hope of prediction, it must emerge from

some more general considerations. Among classes of states which have been studied, those which might be distinguished are:

1. (approximately) supersymmetric states

2. states with large internal volume

3. states exhibiting warping

4. states exhibiting (discrete) symmetries 
While most thinking about states of string theory assumes some approximate supersymmetry, it seems reasonable to think that supersymmetry is special, and that there might be vastly more supersymmetric states than non-supersymmetric states[11, 9, 12, 13]. One can ask, on the other hand, whether cosmologically, such states might be favored. In [15], it was pointed out that primitive cosmological considerations might favor approximately supersymmetric states. Typical stationary points of an effective action are extremely unstable - they are not states at all. Approximately supersymmetric states, however, are inevitably highly metastable. Of the various distinguishing characteristics listed above, apart from supersymmetry, only large volume generically provides stability, and, at least within IIB landscapes, large volume does not appear generic. We won't say more about large volume or warping in this paper, as our considerations don't seem to provide any arguments that these states are special.

In speculating about physics beyond the Standard Model, it has been a widely held belief that discrete symmetries might plausibly - and naturally - solve a range of problems. Such symmetries often arise at special points in the moduli spaces of critical string constructions. But in the framework of the flux landscape, discrete symmetries would seem to be rare[14]. The problem is that the vast number of states purported to exist within the landscape arise because of the existence of a large number of fluxes which can take a large number of values. However, in order to obtain symmetries, many fluxes must be set to zero, greatly reducing the "dimensionality" of the space. In other words, only an exponentially small fraction of states exhibit symmetries.

Again, we can ask whether cosmology might in some way favor (or not) states exhibiting discrete symmetries. Here, the issue is not one of stability, but rather: are the symmetric states, within some realization of eternal inflation, often attractors. We might mean by this that the measure in some inflationary model is highly peaked at the symmetric states, but we will shortly formulate the question in a more naive and primitive way.

Given that there is so much we do not understand about the landscape, the burden of this paper is not to prove that symmetric states are attractors, but rather to establish that this possibility is, or is not, plausible. For this, we seek to adopt models of the states within the landscape, the transitions between them, and the mechanisms by which they are populated. For the states, the challenge is to study the highly non-supersymmetric states which are the antecedents of the would-be states of interest. We will consider a number of models for the states, starting with BP, and then continuing to "states" found as stationary points of supergravity models. These models will incorporate some, but not all, of the features we 
might expect to hold in "realistic" theories. In considering transitions, as we have indicated, we will suppose radii and couplings to be such that these states are states, but that they are highly metastable, so that they do not experience periods of exponential growth. One of our more drastic assumptions will be to treat the fluxes as quasi-continuous. In this way, we can think of tunneling as giving rise to a flow within the landscape, and ask whether, for a given starting point, the system flows towards symmetric states. Such a continuous flux approximation has proven useful in thinking about landscape statistics[16], and has been applied in other cosmological contexts[17]. While surely unrealistic for this problem, it is hard to believe that if symmetries are not attractors in this framework that they are in any realistic setting.

Finally, we need a model for cosmology. Ultimately, we must face up to questions of measures for eternal inflation. But our goal, here, is simply to establish whether there is any reason to think that the rare states in a landscape exhibiting symmetries are somehow favored. For this, again, the burden of this paper is not to establish conclusively that this is the case in an underlying, complete theory of gravity, but simply to establish some general conditions under which symmetries might plausibly be favored. Postulating a landscape with a large number of (very) metastable de Sitter states, we will consider possible "initial conditions" in which the universe starts in one such state. Then we can ask whether, for a non-negligible subset of possible starting points, the system finds its way to the symmetric state. To model this problem, we will suppose that the antecedents of the symmetric state are short lived and do not experience long periods of exponential growth.

In the next section, we lay out certain basic issues involving symmetries in such a picture. We introduce the notion of a neighborhood in the landscape, and argue that it is plausible that an order one fraction of states lie in the neighborhood of states exhibiting discrete symmetries. In section three, we present simple arguments for our basic results: ordinary symmetries are not attractors, but $\mathrm{R}$ symmetries might be. We survey a set of model landscapes in section four. In this discussion, we enumerate some of the present obstacles to developing a landscape cosmology. We discuss the problem of symmetric points within model landscapes. The $T_{6} / Z_{2}$ orientifold is introduced as a model where the symmetry structure is readily understood. Our studies reinforce the arguments of section two, that points exhibiting $R$ symmetries can readily be attractors, but other types of symmetries are not singled out.

In section five, we discuss our conclusions. We explain that these studies are, at best, preliminary and suggestive. Making definitive statements about cosmology certainly requires a deeper understanding of non-supersymmetric configurations within the landscape. But our 
discussion, we believe, makes clear that the viewpoint, advanced by some anthropic advocates (e.g. [18]), that symmetries play no role, does not necessarily follow from adopting a landscape framework. There are clear ways in which supersymmetry and discrete symmetries might well emerge from cosmological considerations. Obviously continuous gauge symmetries are of great interest; we leave their study for future work.

\section{Neighborhoods of Symmetric Points}

States exhibiting discrete symmetries are rare in the flux landscape. But the fraction of states lying nearby such symmetric points need not be small. To understand this, suppose we have some number of symmetric fluxes, $N_{i}, i=1, \ldots I$, and symmetry-violating fluxes, $n_{a}, a=$ $1 \ldots A$. We want to ask whether the set of states in the neighborhood of the symmetric points, i.e., in which $n<N$, is an order one fraction of the total number of states - or more precisely, initial conditions. To model this, let's suppose that the initial state is taken to lie on the "hypersphere"

$$
N_{i}^{2}+n_{a}^{2}=R^{2}
$$

(with summation on $i, a$ ) and ask what fraction of the volume of the sphere satisfies

$$
\alpha^{2} N_{i}^{2}>n_{a}^{2}
$$

For simplicity, we'll take the measure in the space of fluxes to be flat. So we need to compare:

$$
\int d^{I} N d^{A} n \delta\left(\sqrt{N^{2}+n^{2}}-R^{2}\right)
$$

with the same integral, restricted by $n^{2}<\alpha^{2} N^{2}$ (the constant $\alpha$, should be less than one, but our working assumption is that it need not be much less than one). To do the integrals, we need

to recall that the solid angle in $d$ dimensions is: $\Omega_{d}=\frac{2 \pi^{\frac{d}{2}}}{\Gamma(d / 2)}$. So the integral, in the unrestricted case, is simply

$$
\frac{2 R^{I+A-1} \sqrt{\pi}^{I+A}}{\Gamma\left(\frac{I+A}{2}\right)}
$$

With the restriction on the smallness of $n$, the number of states is

$$
\int d^{I} N \int d^{A} n \delta\left(\sqrt{N^{2}+n^{2}}-R^{2}\right) \theta\left(\alpha^{2}\left|N^{2}\right|-\left|n^{2}\right|\right) .
$$




$$
=\frac{2 R^{I+A-1} \sqrt{\pi}^{I+A}}{\Gamma(A / 2) \Gamma(I / 2)} \int_{0}^{\frac{\alpha}{\sqrt{1+\alpha^{2}}}} d x x^{A-1}\left(1-x^{2}\right)^{\frac{I}{2}-1} .
$$

In the case that $\alpha \ll 1$ we can readily estimate the integral, approximating the $(1-x)^{I / 2-1}$ factor in the integrand by 1 . Then the integral behaves as $\alpha^{A}$, while the total number of states behaves roughly as $\left(\frac{A}{2}\right)^{-\frac{I}{2}}$, so the overall suppression is $\alpha^{A}\left(\frac{A}{2}\right)^{\frac{I}{2}}$. For large $A$ and small $\alpha$, this can be very substantial. If $\alpha \simeq 1$, on the other hand, numerical study of the integral indicates that the suppression with increasing $A$ is quite modest. At the moment, the correct value of $\alpha$ is something we can only guess, but we will assume, in what follows, that it is not too small.

One interesting question is how much disfavored are larger symmetries. ${ }^{1}$ As an example, we consider the case, numerically, where we take $\alpha=1$, and compare $I=2, A=10$, with $I=6, A=6$. The difference in the two cases is a factor of 16 , i.e. not huge. So it is not clear that there is an overwhelming preference for smaller symmetries (or at least for small vs. slightly larger symmetries).

\section{Symmetric Points: Attractors Within a Landscape?}

Having established that an order one fraction of states might lie in the neighborhood of a symmetric point, we ask: with our assumptions about initial conditions, is the system more likely to end up in a symmetric state than a non-symmetric state?

Consider, first, the general question of tunneling within a flux landscape. In [15] this question was discussed from a slightly different point of view, which we will adopt here. The general idea is that the large number of states in the flux landscape arise because there are a large number of possible fluxes, $N_{i}=1, \ldots b$, with each of the $N_{i}$ taking on a large number of possible values. The potential, $V(\phi)$, is typically quadratic in fluxes (e.g. in the IIB landscape), $V \sim N^{2}$, so:

1. The difference in energy between states differing by a single unit of flux is of order $N$

2. The tension of the branes separating states differing by a single unit of flux does not depend significantly on the total flux. This follows from considering wrapped branes (NS5, D5). Alternatively, from the point of view of the fields in the low energy theory,

\footnotetext{
${ }^{1}$ We thank L. Susskind for stressing the significance of this issue.
} 
the tension is of order $\sqrt{V(\phi)} \Delta \phi \sim 1$; here $\Delta \phi$, the change in typical fields (e.g. complex structure moduli in the IIB case) is of order $1 / N$.

3. The bounce action for transitions between neighboring states is typically small for large $N$, scaling as

$$
S_{b}(\Delta N=1) \sim \frac{1}{N^{3}}
$$

Ref. [15] focussed on the question: if a state happens to have small cosmological constant, is it stable against decay to its numerous neighbors with negative cosmological constant. Here we are asking a different sort of question. We are studying the ancestors of the symmetric states. Since we are interested in the symmetric states as candidate theories of nature, we will assume that they have small cosmological constant; the ancestors have positive cosmological constant. The estimate above indicates that these states are typically highly unstable - they are not states at all. But, as a model, we will take this type of formula literally (one can suppose, for example, that the states, for some reason, all have moderately large volume, enhancing the bounce action). In the same spirit, we will treat the fluxes as large enough that they can be treated as quasi-continuous[16]. In such a picture, there is a well-defined notion of neighborhood in the landscape.

As a further, rather drastic simplification, we will ignore gravity in estimating decay amplitudes. This is often valid (as discussed, for example, in [2]), for some range of fluxes and values of moduli, but it is not likely to hold generally. For example, among the many numerous metastable dS states, it is likely that some are long-lived, giving rise to (eternal) inflation. As we will see, even with such drastic assumptions, only in a limited set of instances do symmetric states seem singled out by cosmological considerations.

\subsection{Non-Symmetric States}

Suppose that the putative state of interest, with small cosmological constant, lies at some point in the flux lattice $\vec{N}_{0}$. In this case, in the continuous flux approximation, we would expect the energy, near that point, to have the form:

$$
E_{0}=C_{a} \Delta N^{a}
$$

Recall the basic assumption that there is a large, multi-dimensional space of states. So, from the tunneling perspective, with our assumption about initial conditions, it is clear that from 
most starting points, one will "miss" the state $\vec{N}_{0}$, ending up eventually tunneling to negative cosmological constant big crunches. The chance of ending up in the "correct vacuum" will be of order one divided by the number of low cosmological constant states.

\subsection{The neighborhood of symmetric states}

Consider a state which is symmetric or approximately symmetric under an ordinary discrete symmetry. Some subset of fluxes, $N_{i}, i=1, \ldots, B$, respect the symmetry (they are neutral under the symmetry, and there are minima of the resulting potential in which only fields neutral under the symmetry have expectation values). The rest, $n_{a}, a=1, \ldots, A$, break the symmetry. The putative, low cosmological constant, symmetric state we denote, again, by $\vec{N}_{0}$. The set of states with flux

$$
\left|n_{a}\right|<\left|N_{i}-N_{i}^{0}\right|
$$

define the neighborhood of the symmetric point. We will take $n$ to denote an average $n_{a}, N$ an average $N_{i}$.

If we assume that in the symmetric state there are no moduli, then in the continuous flux approximation, the energy landscape nearby has the form

$$
E_{0}=\sum_{i, J=1}^{I} f_{I J} N_{I} N_{J}+\sum_{a, b=1}^{A} g_{a b} n_{a} n_{b}
$$

Again, in the continuous flux approximation, we can diagonalize $g$. We can immediately distinguish two cases.

1. $g_{a b}$ has only positive eigenvalues. In this case, if one starts in the neighborhood of the symmetric state, at least the transitions which change the $n_{a}$ 's will tend towards the symmetric state. A priori, we might simply assign a probability $1 / 2$ for the possibility that a particular eigenvalue is negative, so there is (compared to factors like $10^{500}$ ) a modest suppression of this possibility.

2. $g_{a b}$ has some negative eigenvalues. In this case, the corresponding $n$ 's will tend to grow, and the system will not tend towards the symmetric state.

But even in the case where all of the eigenvalues of $g$ are positive, there is nothing particularly special about transitions to the symmetric $\vec{n}_{a}=0$ state. Assuming the eigenvalues of $g$ 
are all similar, if $n_{a} \ll N_{i}$, then

$$
\frac{S_{b}\left(\delta n_{a}=1\right)}{S_{b}\left(\delta N_{i}=1\right)} \sim \frac{N^{3}}{n^{3}}
$$

So transitions which change $n$ are much slower than those which change $N$. Under such circumstances, the notion of a neighborhood is not particularly relevant to the tunneling process. Unless one starts in the state with $n_{a}=0$, one will not reach the symmetric states; one may reach a state $\left(\vec{N}_{0}, \vec{n}_{a}\right)$, but then one will transition to big crunches with negative cc.

Suppose, on the other hand, that the elements of $g$, in addition to being positive, were far larger that those of $f$. Then transitions involving changes in $n$ would be faster than those involving changes in $N$, and states in the neighborhood would, preferentially, end up in the symmetric state. Such a situation could conceivably arise if there were approximate moduli in the symmetric state. Then, in the nearby states, some quantities (volumes of cycles, for example) might be quite different than in the symmetric state, and tunneling rates could be quite different. We will a phenomenon of this sort in the case of $\mathrm{R}$ symmetric vacua.

\subsection{R Symmetries}

Let's turn our attention to $R$ symmetric states. Here there is an important difference with the non-R case. Changes in $N$, in the symmetric limit, are not associated with changes in energy, so they are potentially highly suppressed.

To go further, we need to think more carefully about the $\mathrm{R}$ symmetric states. In general, suppose that we have fields, $X_{I}$, which transform under the $R$ symmetry like the superpotential, and fields, $\phi_{\alpha}$, which are neutral. There are additional fields, $\rho_{i}$, which transform differently than the $X$ 's under the $R$ symmetry. Then the superpotential has the form

$$
W=X_{I} F_{I}\left(\phi_{\alpha}, \rho_{i}\right)
$$

There will be supersymmetric, $R$ symmetric solutions $(X=\rho=0)$ provided there are more $\phi$ type fields than $X$ type fields. In this case, in general, there will be some number of moduli. This condition holds in many Calabi-Yau compactifications, for example[14].

In the present context, this means that classically the vacua with $n_{a}=0$ possess moduli. Our working assumption will be that these moduli are fixed by non-perturbative dynamics, as discussed in [19], with supersymmetry broken at comparatively low scales. For non-zero $n_{a}$, the situation is quite different. We can study an effective action for the moduli. Their potential is 
of order $n^{2}$. Correspondingly, the masses-squared of the light moduli are of order $n^{2}$. As we will see in model examples shortly, the corresponding ground states can be dS or AdS. If much of the neighborhood of the symmetric point is AdS, we don't expect a significant probability to reach the symmetric point. So we will assume that the neighbors of the symmetric point are predominantly $\mathrm{dS}$.

In order to compare tunneling rates for processes with changes in $N$ with those with changes in $n$, we need to understand how the properties of the lagrangian for the light moduli depend on $N$. We expect that this dependence is quite weak; indeed, we might guess that it goes as $n^{4} / N^{2}$. We can illustrate the point, again, with a simple field theory model. Consider a theory with three fields, $X$ and $\phi, \chi$, where $X$ transforms like $W$ and $\phi, \chi$, are neutral. For the superpotential we take

$$
W=\lambda X(\phi+\chi)(\phi-\chi)
$$

(the precise dependence on $\phi$ and $\chi$ is not crucial, but makes the equations simple). There is a moduli space, on one branch of which

$$
\phi=\chi=v
$$

On this branch, there are two massive fields, $X$ and $\Phi=\frac{1}{\sqrt{2}}(\phi-\chi)$, and one massless field, $\psi=\frac{1}{\sqrt{2}}(\phi+\chi)$. Now, the effect of some underlying theory in which we turn on fluxes, $n_{i}$, would be to generate a superpotential of the form, say

$$
\Delta W=\epsilon(\alpha+\beta \psi+\gamma \Phi) .
$$

where $\epsilon \sim n$. Such a potential can fix the remaining modulus (and break supersymmetry). It also generates a tadpole for $\Phi$, of order $\epsilon^{2}$, and a $\lambda$-dependent term in the energy of order

$$
\Delta E \approx \frac{\epsilon^{4}}{\lambda^{2} v^{2}} \sim \frac{n^{4}}{N^{2}} .
$$

We will see some of these features in more detailed models in subsequent sections.

We will be interested in the relative size of transition rates for changes in $n$ and changes in $N$. We have understood the energy splittings between the different types of states; we need to ask about the tensions. One might think that the tensions for changes in $N$ would also be suppressed, as, for BPS configurations, the tension is proportional to $\Delta W$. But already in field theory, domain walls separating $R$ symmetric states are not BPS. As a simple example, consider a theory with superpotential

$$
W=X\left(\phi^{2}-\mu^{2}\right)
$$


This model has an $R$ symmetry and a $Z_{2}$ symmetry. The states $\phi= \pm \mu$ are separated by a region in which $\phi \neq 0$, and $\frac{\partial W}{\partial X} \neq 0$, but in which $\partial_{\mu} X=0$, so they are not BPS, and their tension is certainly non-vanishing. In the flux case, we expect, again, that the tension is not very sensitive to $N$. So the bounce action for changes in $N$ is larger than that for changes in $n$ by

$$
\frac{S_{b}(\Delta N=1)}{S_{b}(\Delta n=1)}=\frac{N^{9}}{n^{9}} !
$$

So in this case, transitions towards the symmetric point are likely to be much faster than other transitions.

This discussion, while suggestive, is not complete. Much depends on the states in the low energy theory, defined by the $n_{i}$ 's. For example, this theory may itself have many AdS states, as we will see, which can mean that the system does not reach the symmetric point. We will discuss this issue in the models of the subsequent sections. Still, we see a sense in which symmetric points, and especially $\mathrm{R}$ symmetric points, are special, and could plausibly be attractors in a flux landscape.

\section{Model Landscapes}

We are far from understanding the structure of any realistic landscape. We will review some of the reasons for this shortly, but just note for now that this means that any understanding we have of the landscape - especially in a cosmological setting - comes from consideration of model landscapes, which it is hoped capture some of the important features of what might be the "real" situation.

\subsection{The Bousso-Polchinski Model}

The first compelling model of a landscape was that of Bousso and Polchinski (BP)[2]. This was based on consideration of four-form fluxes in Type II theories. One notable feature of the model is the assumption that all moduli are fixed for any choice of fluxes, in every state (and fixed, essentially to the same value). The energy spectrum is taken to have the form:

$$
E_{0}=\frac{1}{2} N_{i}^{2} q_{i}^{2}-\Lambda_{0}
$$

The $q_{i}$ 's are constants, independent of $N_{i}$. They are assumed to be small enough that all tunneling amplitudes are small. This requires (in accord with the estimates in [15]) that the 
internal manifold be large, with volume scaling as a positive power of the flux. This model is extremely useful, first, for illustrating the idea of a discretuum: the model exhibits a nearly continuous distribution of energies for large fluxes. It also provides a model for eternal inflation.

With these assumptions, if the $q_{i}$ 's are comparable, transitions tend to decrease the fluxes uniformly. One can make a rough estimate of the transition rate by ignoring gravity and using the formulas appropriate to the thin wall limit[20,21]. This was done in [2], in the limit that the compactification volume and the radii of various three cycles are large. The spirit of our analysis is to consider the case that radii are of order the fundamental scale, and to examine scaling with $N$. Then the bubble wall tensions are of order 1, while the energy splitting between states is of order $N$. So the bounce action behaves as $1 / N^{3}$. As noted in [2], even moderately large volume can significantly suppress the tunneling rate. Note that if one of the $N_{i}$ 's becomes much smaller than the others, further transitions in that flux are suppressed, i.e. all fluxes tend to decrease uniformly.

\subsection{IIB Compactifications: Limitations of the BP Model and The Continu- ous Flux Approximation}

Known flux models differ from that of BP in some significant ways. First, when moduli are fixed, they are typically non-trivial functions of the fluxes (this, and related points, have been made in [17]). In the IIB theory, for example, the potential for the moduli assumes the usual $\mathcal{N}=1$ supergravity form:

$$
V=e^{K}\left(g^{i \bar{j}} D_{i} W \overline{D_{j} W}-3|W|^{2}\right)
$$

where $i, j$ are indices labeling the dilaton, complex structure and Kahler moduli. The superpotential, $W$, is linear in the fluxes, so

$$
V=N_{i} N_{j} f_{i j}\left(\phi_{\alpha}\right)
$$

where $N_{i}, N_{j}$ are the fluxes and $\phi_{\alpha}$ denote the various fields. If we can think of all fluxes as large, it is natural to write:

$$
N_{i}=N u_{i}
$$

Then, at stationary points of the potential, the $\phi_{\alpha}$ are independent of $N$, and the potential is proportional to $N^{2}$. But both $\phi_{\alpha}$, and $V_{0}$, the values of the potential at the stationary points, are dependent on the $u_{i}$ 's. We will illustrate this with some simple toy models shortly. 
In the IIB models, there is another important difference: there are no flux-independent terms in the classical potential derived from the supergravity lagrangian. As a result, there is no obvious identification of the parameter $\Lambda^{2}$ or positivity of the parameters $q_{i}^{2}$, and no clear sense in which the fluxes should tend uniformly towards zero with successive tunneling transitions.

But perhaps the most crucial difference is related to the Kahler moduli (which we will denote generally by $\rho$ ). The potential for the complex structure moduli has stationary points, but at large radius of the internal manifold, there are no stationary points for $\rho$; both perturbatively and non-perturbatively, the potential tends to zero for large $\rho$. As a result, the system is prone to run off to large radius. The value of $\rho$, and of the potential (and other quantities) is not similar at the KKLT point and at adjacent points. There may be stationary points (metastable vacua of the full quantum system) at small $\rho$ (i.e. $\rho \sim 1$ ) for some or most choices of flux (or slightly larger $\rho$, as in KKLT, see below), but one would expect that if one starts in a high cc state with all moduli fixed, one is likely to tunnel to a configuration which simply rolls out to large $\rho$. Understanding the significance of this (assuming it has a sensible interpretation) probably requires a deeper understanding of eternal inflation, and especially how states are populated.

\subsection{KKLT and Limitations of the Continuous Flux Approximation}

The KKLT model has become a paradigm for the problem of fixing moduli in flux vacua. While one can raise issues about the analysis (i.e. in what sense the approximations are truly systematic), it provides a plausible scenario for fixing of moduli in supersymmetric and approximately supersymmetric IIB compactifications. It also provides an illustration of the ways in which realistic landscape states are unlike the BP model and similar theories which have been used to consider eternal inflation and related issues.

The basic ingredients in KKLT are a IIB theory compactified on a CY Manifold (Orientifold). Before turning on fluxes, the light fields of such a compactification consist of complex structure moduli and dilaton $\left(z_{i}, \tau\right)$, and Kahler moduli $\left(\rho_{a}\right)$. Fluxes yield a superpotential $W(z, \tau)$. The equations $D_{z_{i}} W=0, D_{\tau} W=0$, in general, fix $z_{i}, \tau$.

For large $\rho$, the low energy effective theory consists of the Kahler moduli. In the case of a single Kahler modulus, KKLT argue that the superpotential and the Kahler potential have the form:

$$
W=W_{0}+e^{-b \rho} ; \quad K=-3 \ln \left(\rho+\rho^{\dagger}\right)
$$


If there are many states, then, in some, KKLT argued that $W_{0}$ will be small; in these, the effective action gives rise to an AdS, supersymmetric minimum with $\rho \sim-\frac{1}{b} \ln \left(W_{0}\right)$. Supersymmetry, they argued, could be broken by antibranes; alternatively, dynamics of low energy gauge interactions might provide a breaking mechanism.

The KKLT model makes especially clear the limitations of the continuous flux approximation. The features of KKLT depend crucially on the smallness of $W_{0}$, which relies on cancelations of fluxes. For nearby choices of flux, $W_{0}$ is order one in fundamental units. So in this instance, we need to step away from the continuous flux approximation, and consider what the neighboring states look like. These states might then be expected to have broken supersymmetry, with $\rho$ fixed, if at all, at some high energy scale, and cosmological constant (positive or negative) also very large. Moreover, in all of these states, the potential vanishes for very large $\rho$. So how transitions might proceed towards the KKLT vacua is not clear. Transitions from relatively nearby states, might be expected to end, typically, in big crunches or with the universe rolling - eternally - to large $\rho$. It could well be that anthropic considerations permit one to ignore these "bad ends", but without a larger theoretical framework, it seems like this will be a challenging story to sort through.

For the $R$-symmetric vacua that we consider shortly, the situation may often be different. Configurations starting out in a finite neighborhood may well end up in the preferred state a finite fraction of the time.

\subsection{The Polonyi Model}

Setting aside the problem of $\rho$, if we consider models which capture more of the features of the IIB theory, we encounter a richer and more subtle structure. Consider, first, a familiar example, the Polonyi model. We will think of the parameters of the superpotential of this model as surrogates for flux (we will shortly consider compactifications with a similar structure, where these parameters are determined by fluxes). The model consists of a singlet chiral field $z$, with superpotential:

$$
W=\alpha+\beta z ; \quad K=z z^{*}
$$

As one varies the parameters, this has both supersymmetric and non-supersymmetric minima. In the supersymmetric minima, $\langle W\rangle$ is not a simple linear function of the fluxes.

$$
z=\frac{-\alpha \pm \sqrt{\alpha^{2}-4 \beta^{2}}}{2 \beta} ; \quad W_{0}=\frac{\alpha \pm \sqrt{\alpha^{2}-4 \beta^{2}}}{2}
$$


For large $\beta$, there are no supersymmetric solutions. The non-analytic behavior as a function of the fluxes is not surprising. If $\alpha=\beta=0$, there is a moduli space of vacua. Turning on the fluxes lifts the degeneracy. This phenomenon is analogous to phenomena in ordinary degenerate perturbation theory, in which non-analyticity in an expansion parameter is typical.

The non-supersymmetric branch exhibits similar behaviors. We can rewrite the superpotential as

$$
W=\alpha(1+u z)
$$

with $u=\beta / \alpha$; $\alpha$ then plays the role of $n$ in our earlier discussions, and $u$ of one of the "angles" introduced there. To simplify the algebra, we will assume that $\alpha$ and $\beta$ are real; we will also assume that the vev of $z,\langle z\rangle=x$ is real (this preserves a CP symmetry of the lagrangian). Then the potential is

$$
V(x)=\alpha^{2} e^{x^{2}}\left(u^{2}\left(x^{4}-x^{2}+1\right)+2 u\left(x^{3}-2 x\right)+x^{2}-3\right) .
$$

The equation, $\frac{\partial V}{\partial x}=0$ then takes the form:

$$
u^{2}\left(x^{5}+x^{3}\right)+u\left(2 x^{4}-x^{2}-2\right)+x^{3}-2 x=0 .
$$

Solving for $x$ in terms of $u$ is difficult, but solving for $u$ in terms of $x$ is simple. One root is the supersymmetric branch we discussed above:

$$
u=-\frac{x}{1+x^{2}}
$$

The second, non-supersymmetric branch has:

$$
u=-\frac{x^{2}-2}{x^{3}} .
$$

We focus here on the non-supersymmetric branch. Substituting $u$ into the potential, yields the value of the energy at the minimum of the potential for a given $x$ :

$$
V_{0}=\alpha^{2} \frac{e^{x^{2}}}{x^{6}}\left[x^{4}-8 x^{2}+4\right] \text {. }
$$

Note that the vacuum energy is positive for:

$$
x^{2}>4+2 \sqrt{3} \quad x^{2}<4-2 \sqrt{3} .
$$

We need to check stability; a little bit of algebra yields::

$$
\frac{d^{2} V}{d x^{2}} \propto \frac{1}{x^{6}}\left[-x^{6}+4 x^{4}+12 x^{2}\right] .
$$


Note that this is positive for sufficiently small $x$, and badly behaved for large $x$. So we have established the existence of a range of parameters for which there are stable stationary points where the vacuum energy is positive and supersymmetry is broken.

For large and small $u$, we can solve the equations. In both limits,

$$
u=-\frac{1}{x} \quad x=-\frac{\alpha}{\beta}
$$

For small $\beta$ (large $x$ ), the vacuum energy is approximately

$$
V=\beta^{2} e^{\alpha^{2} / \beta^{2}}
$$

while for small $\alpha$ (small $\mathrm{x}$ )

$$
V=4 \beta^{6} / \alpha^{4}
$$

We see that the neighborhood of the $\alpha=\beta=0$ point is surrounded by a sea of negative cosmological constant states with $\alpha$ and $\beta$ comparable.

Thinking of $\alpha$ and $\beta$ as fluxes, we will take these parameters as large integer multiples of some basic unit. We will estimate tunneling rates, as in [15], by assuming that the tension is proportional to $\Delta x$ in the transition, and the bounce action is proportional to

$$
S_{b} \sim T^{4} /(\Delta V)^{3}
$$

Then an interesting question to ask is: starting in a configuration with $\alpha \ll \beta$, does the system flow towards the region of AdS states with $\alpha \approx \beta$, or does it flow towards the zero energy states with $\alpha=0$. Estimating the bounce action as above, it is easy to see that

$$
\frac{S_{b}(\Delta \alpha=1)}{S_{b}(\Delta \beta=1)}=\frac{\beta}{\alpha}
$$

i.e. the transitions which decrease $\beta$ are faster than those which decrease $\alpha$. The couplings tend to equalize, and one is driven to the negative c.c. regime.

While a simple, toy, model, the Polonyi model already captures certain features of the IIB flux vacua. In particular, the expectation values of moduli fields are complicated functions of the fluxes (both for supersymmetric and non-supersymmetric vacua). As a result, the tunneling behavior of the system is complex, with the system, for example, tending to avoid the zero c.c. region and heading rather to a region with negative cosmological constant. We will encounter behaviors of this type in a more intricate model in the next section. 


\subsection{Multiple-Field Polonyi Model}

In the landscape framework, we are interested in models with many fields, analogs of the field $z$. We can ask whether there are typically mainly dS or AdS vacua. A model which is simple to analyze has $P$ fields and a $S_{P}$ symmetry:

$$
W=a \sum_{i} z_{i}+b
$$

To make the analysis simple we will:

1. Look for stationary points where the $S_{P}$ symmetry, and CP, are preserved, i.e. $z_{i}=x$.

2. Take the Kahler potential to be $z_{i}^{*} z_{i}$, and ignore the $e^{K}$ terms in the potential.

We will study the potential in the limit of large $P$. In this limit, even though the resulting equations are cubic, we will be able to construct a solution in powers of $1 / \sqrt{P}$. The potential is:

$$
V=\sum_{j}\left(a+z_{j}^{*} W\right)\left(a+z_{j} W^{*}\right)-3 W^{*} W
$$

so

$$
\frac{\partial V}{\partial z_{i}^{*}}=W\left(a+z_{i} W^{*}\right)+\sum_{j}\left(a+z_{j}^{*} W\right) z_{j} \frac{\partial W^{*}}{\partial z_{i}^{*}}-3 W \frac{\partial W}{\partial z_{i}^{*}}
$$

At the would-be minimum,

$$
W=a P x+b .
$$

So, substituting above, we obtain

$$
0=P^{2}\left(2 a^{2} x^{3}\right)+P\left(3 a b x^{2}-a^{2} x\right)+\left(b^{2} x-2 a b\right)
$$

For large $P$, in order to solve this equation, it is necessary that $x$ take the form:

$$
x=\frac{\alpha}{P^{1 / 2}}+\frac{\beta}{P}+\ldots
$$

It is easy to see that $\alpha=\frac{1}{\sqrt{2}}$. As a result, at the minimum,

$$
W \approx \frac{a}{\sqrt{2}} P^{1 / 2}
$$


On the other hand,

$$
D W \approx a+\frac{1}{\sqrt{2 P}} \frac{a}{\sqrt{2}} P^{1 / 2} \approx \frac{3}{2} a
$$

so the potential is:

$$
V=\sum|D W|^{2}-3|W|^{2} \approx \frac{3}{4} a^{2} P
$$

So the solutions are De Sitter, independent of $a$ and $b$. Including $e^{K}$, one finds, for large $P$, that the minima lie near the origin, with cosmological constant

$$
\Lambda \approx P a^{2}
$$

Similar results hold for other choices of Kahler potential, such as $K=-\ln \left(1+z_{i}^{*} z_{i}\right)$.

Clearly this is just a model, and one could well imagine that other parameter choices (and possibly other stationary points) would yield AdS minima, but this illustrates that there is no particular problem, with large numbers of fields, obtaining dS spaces.

\subsection{General Orientifolds of IIB on Calabi-Yau Spaces}

In order to relax some of the assumptions of the BP model, it is useful to consider flux vacua within the context of actual string compactifications in which the fixing of moduli is more or less understood. Perhaps the most widely studied flux vacua are provided by orientifolds of IIB theories on Calabi-Yau spaces. Here the candidate fluxes are RR and NS-NS three form fluxes. These are constrained by tadpole cancelation conditions. Most analyses have focussed on a low energy supersymmetric effective lagrangian, obtained by reduction of the ten dimensional supergravity theory. This lagrangian is described by a Kahler potential and superpotential for "light" fields. There are two classes of moduli which must be understood: complex structure moduli, $z_{i}$, and Kahler moduli, $\rho_{a}$. Classically, the superpotential depends only on the $z_{i}$ fields. Typically, one first tries to solve the equations for a supersymmetric stationary point with respect to the complex structure moduli, $D_{z_{i}} W=0$. The Kahler moduli yield a no-scale structure, with vanishing potential. If the solutions of the equations $D_{z_{i}} W=0$ yield $W=0$, then the classical system is fully supersymmetric. One might expect, for complicated models, that $W=0$ is the result of an (unbroken) discrete $\mathrm{R}$ symmetry.

Our interest, for modeling transitions in the landscape, lies in non-supersymmetric solu-

tions. There are two approaches one might adopt, closely related to analyses of Douglas and Denef[9]. In one, we will discard the Kahler moduli, keeping only the complex structure moduli. 
In this analysis, non-susy states are stationary points of the potential, for which $D_{i} W \neq 0$. For most choices of flux, there are, indeed, no supersymmetric solutions. These models resemble the Polonyi model, with many AdS minima. In the second, we keep the Kahler moduli, using the lowest order Kahler potential, and assuming that the Kahler moduli are somehow fixed at large values. SUSY is broken in all of these states, which are either dS or flat.

\section{7 $\quad T_{6}$ Inspired Model}

Perhaps the simplest example of this type is the orientifold of the IIB theory on a $T_{6}$ lattice, studied by [22]. This theory is described by a set of complex structure moduli, $\tau_{i j}$. Correspondingly, there are a set of NS-NS and R-R fluxes. In this section, we will consider a particular limit of this orientifold, which will allow us to illustrate certain basic issues surrounding symmetries. Our principle interest is in non-supersymmetric, dS vacua, which we will not be able to describe, in general, in any systematic way. So we will adopt a simple model. We will take the radius to be large and fixed, and examine the potential for the complex structure moduli, ignoring the dilaton. At the same time, we will include only the NS-NS fluxes. In this case the fields of the model are $\tau_{i j}, i, j=1, \ldots 3$. They are described by a superpotential:

$$
W=a_{0} \operatorname{det}(\tau)+a_{i j}(\operatorname{cof}(\tau))_{i j}+b_{i j} \tau_{i j}+b_{0}
$$

and a Kahler potential

$$
K=\ln \left(i \operatorname{det}\left(\tau-\tau^{\dagger}\right)\right)
$$

For special choices of the fluxes, the resulting lagrangian exhibits global symmetries. For example, for

$$
a_{i j}=a_{1} \delta_{i j} \quad b_{i j}=b_{1} \delta_{i j}
$$

the lagrangian possesses an $S U(3)$ symmetry. This symmetry is broken by the underlying lattice to, at most, some discrete subgroup (we will discuss the symmetries of this model, in general, shortly), but this breaking is not visible in the classical theory. As we will see, such a choice of flux is enough to fix all of the moduli.

To summarize the model:

1. We ignore the dilaton 
2. We keep the Kahler moduli, $\rho_{i}$, but assume that they are fixed at some large value, where they can be described by their classical Kahler potential, and $W$ is independent of $\rho$. The effect of this is to cancel the $3|W|^{2}$ in the lagrangian for the $\tau_{i j}$ 's.

3. We work in the continuous flux approximation, ignoring tadpole cancelation constraints.

Even with these simplifications, the potential for the moduli is rather complicated to analyze. In certain limits, however, it is simple. An easy case to study is that with $b_{0}=0$, and $a_{1}, b_{1}$ proportional to the unit matrix. With $\tau=i x$, the potential is given by

$$
V=\frac{3\left(a_{1}^{2} x^{2}+\left(b_{1}+a_{0} x^{2}\right)^{2}\right)}{8 x}
$$

which has a minimum for

$$
a_{1}=\sqrt{\frac{\left(b_{1}-3 a_{0} x^{2}\right)\left(b_{1}+a_{0} x^{2}\right)}{x^{2}}} .
$$

At this minimum, one can check that there are no tachyons and the potential is positive provided

$$
-b_{1}<a_{0} x^{2}<-\frac{6 b_{1}}{17+\sqrt{145}}
$$

To understand the significance of this condition, it is useful to express $x^{2}$ in terms of $a_{1}, b_{1}, a_{0}$.

$$
x^{2}=\frac{-\left(a_{1}^{2}+2 a_{0} b_{1}\right) \pm \sqrt{\left(a_{1}^{2}+2 a_{0} b_{1}\right)^{2}+12 a_{0}^{2} b_{1}^{2}}}{6 a_{0}^{2}} .
$$

\subsection{Symmetries of the $T_{6}$ Model}

Before turning on fluxes, the $T_{6}$ model has a large moduli space. On various subspaces, it exhibits symmetries. For example, for $\tau$ proportional to the unit matrix, one has separate $Z_{4}$ symmetries in each of three planes, as well as an $S_{3}$. In terms of the underlying (real) coordinates, $x^{i}, y^{i}, i=1, \ldots, 3$, the $Z_{4}$ 's take

$$
x^{i} \rightarrow y^{i} \quad y^{i} \rightarrow-x^{i}
$$

( $i$ fixed). These are $R$ symmetries, because they change the sign of the holomorphic three form,

$$
d \Omega=d z^{1} \wedge d z^{2} \wedge d z^{3}
$$

with

$$
z^{i}=x^{i}+\tau_{i j} y^{j}
$$


Odd permutations are also $R$ symmetries.

But we need to ask, as well, how the fluxes transform under the various symmetries. Ref. [22] provides a convenient catalog of three forms $\left(H_{3}\left(T_{6}, Z\right)\right)$ :

$$
\begin{gathered}
\alpha_{0}=d x^{1} \wedge d x^{2} \wedge d x^{3} \quad \beta_{0}=d y^{1} \wedge d y^{2} \wedge d y^{3} \\
\alpha_{i j}=\frac{1}{2} \epsilon_{i k \ell} d x^{k} \wedge d x^{\ell} \wedge d y^{j} \beta^{i j}=-\frac{1}{2} \epsilon_{j k \ell} d y^{k} \wedge d y^{\ell} \wedge d x^{i}
\end{gathered}
$$

The corresponding fluxes are the quantities $a_{0}, b_{0}, a^{i j}, b_{i j}$ encountered above.

It is easy to read off the transformation properties of the fluxes from these expressions under the various symmetries. Our particular choice, $a_{i j}=a_{1} \delta_{i j}$, breaks all of the $R$ symmetries. It leaves over cyclic transformations of the coordinates (i.e. even permutations).

Now consider turning on small off-diagonal entries in $a_{1}, b_{1}$, breaking the microscopic cyclic symmetry (in the lagrangian itself, these break the $S U(3)$ symmetry). For small $a_{1}$, positive $b_{1}$ and negative $a_{0}$, the condition, eqn. 51 , for the absence of tachyons, reads, approximately:

$$
-b_{1}<-\frac{b_{1}}{3}<-b_{1} / 5
$$

which is always satisfied. Because there are no tachyons, this generates shifts in $\tau_{i j}$ of order $\delta a_{1}, \delta b_{1}$, and changes in the energy of order $\delta a_{1}^{2}, \delta b_{1}^{2}$. Transitions between states are associated with five branes wrapping three cycles. If radii are of order one, then the tensions are of order one (i.e. they are not enhanced by powers of flux). So, for small $\Delta a_{1}, \Delta b_{1}$, transitions towards the symmetry point are slow; there is no particular tendency of the system to flow towards the symmetry point; only for very special initial conditions is symmetry achieved.

\subsection{R Symmetries within the $T_{6}$ Model}

A simple example of an $\mathrm{R}$ symmetry in the $T_{6}$ model is provided by taking a lattice which is a product of three square lattices in each of three planes. Consider the symmetry:

$$
x_{1} \rightarrow y_{1} \quad y_{1} \rightarrow-x_{1}
$$

This commutes with the orientifold symmetry. We can classify fluxes according to their behavior under this symmetry. The invariant three forms are:

$$
\alpha_{1 a} \quad \beta^{a 1}
$$


and

$$
\alpha_{a 1} \quad \beta^{1 a}
$$

where $a=2,3$. The fields $\tau_{1 a}, \tau_{a 1}$ transform like the superpotential, i.e. by a phase $e^{\frac{2 \pi i}{4}}$. With $\tau_{11}=i+\delta \tau, \delta \tau \rightarrow-\delta \tau$ under the symmetry.

To make our expressions simple, we will also impose a symmetry $2 \leftrightarrow 3$ on the fluxes. Then calling the fluxes (as in [22]) $a_{1 a}, b_{a 1}$, etc ,the superpotential is:

$$
\begin{aligned}
W & =a_{12}\left[\tau_{21} \tau_{33}-\tau_{23} \tau_{31}+\tau_{31} \tau_{22}-\tau_{32} \tau_{21}\right]+a_{21}\left[\tau_{12} \tau_{33}-\tau_{13} \tau_{32}+\tau_{13} \tau_{22}-\tau_{23} \tau_{12}\right]+ \\
& +\quad b^{12}\left[\tau_{12}+\tau_{13}\right]+b^{21}\left[\tau_{21}+\tau_{23}\right]
\end{aligned}
$$

We are looking for supersymmetric stationary points with vanishing $W$, so we wish to solve

$$
\frac{\partial W}{\partial \tau_{i j}}=0
$$

with $\tau_{11}=\tau_{a 1}=\tau_{1 a}=0$. For general choices of the fluxes $a_{1 a}$, etc., the resulting equations, however, are incompatible. In the language we used in section 3.3, there are more $X$ type fields $\left(\tau_{1 a}, \tau_{a 1}\right)$ than $\phi$ type fields $\left(\tau_{22}, \tau_{33}\right)$. In more elaborate models (e.g. Calabi Yau's defined by intersections in weighted projective space) this is not the case. In the present model, solutions do exist for special choices of flux. In particular, if we take

$$
b^{12}=b^{21} \quad a_{12}=a_{21}
$$

then there is a moduli space with

$$
\tau_{22}=\tau_{33} \quad \tau_{23}=\tau_{32}=\tau_{33}+\frac{b_{12}}{a_{12}}
$$

The next step is to turn on small symmetry violating fluxes, fixing these remaining moduli, and studying the behavior of the energy and fields as functions of the small fluxes. To first approximation, we simply need to write the effective action for the light moduli, and find its stationary points. There are many fluxes which can be turned on, and the resulting potentials, for which supergravity corrections are important, are complicated. To get some flavor for the problem, note that we need to fix $\tau_{11}, \tau_{22}$ and $\tau_{33}$. So we can turn on small fluxes $a_{0}$, and similarly $b_{11}, b_{22}, b_{33}$ (again, to keep things simple, we will impose a $1 \leftrightarrow 2$ symmetry). Then the added terms in the superpotential are:

$$
W_{l . e .}=a_{0}\left(\tau_{11} \tau_{22} \tau_{33}-\tau_{11} \tau_{23} \tau_{32}+\ldots\right)+b_{11} \tau_{11}+b_{22}\left(\tau_{22}+\tau_{33}\right) .
$$


One can check that, with these choices, there are no tadpoles for $\tau_{1 a}, a=1,2$. We can substitute for $\tau_{23}, \tau_{32}$ their forms on the moduli space, eqn. 63. The resulting expression is similar to that we studied in section 4.7, and we expect a similar structure for the solutions. Energies are of order $n^{2}$ (where the $n$ 's are the symmetry-breaking fluxes above). In particular, we expect that the diagonal elements of $\tau$ are fixed, that for a range of fluxes there are no tachyons, and that transitions tend to keep the diagonal elements of $a, b$ comparable. So shifts of the heavy fields will be of order $n^{2} / N^{2}$.

Now we see that the tunneling rates are as we guessed for $R$-symmetric stations in section 3. Energies have the form:

$$
E_{0}=a n^{2}+b \frac{n^{4}}{N^{2}}
$$

so energy splittings between states of different $N$ behave as $n^{4} / N^{3}$. The tensions, again, are of order one, parametrically, the bounce actions for transitions which change $N$ behave as

$$
S_{b}(\Delta N=1) \approx \frac{N^{9}}{n^{12}}
$$

On the other hand, transitions which change $n$ simply scale as

$$
S_{b}(\delta n=1) \approx \frac{1}{n^{3}}
$$

So symmetry-restoring transitions are highly favored.

\section{Conclusions}

Even if underlying fundamental physics is some type of landscape of metastable vacua, it is unlikely that, in the near future, we will have anything resembling a complete catalog of these states. Instead, any sort of understanding of the connection of microphysics and the phenomenon we observe will have to be based on robust statements about distinctive features of physics at much lower energy scales. In an earlier paper[15], we focussed on supersymmetry and the question of stability. In the present paper, we have mainly considered discrete symmetries. We have remarked that states with discrete symmetries are rare[14], but suggested that they might be cosmological attractors. This was motivated by the naive assumption that the potential might grow quadratically with flux around the symmetric point, so the system, starting in the neighborhood of the symmetric point, might tend to tunnel towards the symmetric point. To study this problem we have considered several model landscapes, which capture likely 
features of a would-be underlying cosmic landscape. Even before approaching the problem of symmetries, we have argued that the Bousso-Polchinski model (the simplest model landscape we have considered) is likely too simple a picture, given its assumptions about positivity and constancy of flux coefficients, and given the existence of asymptotic pseudomoduli spaces. We have considered a number of toy models which capture more, but not all, of these expected features.

To consider the question of whether symmetric points are attractors, we have worked in the continuous flux approximation. This is hardly likely to be realistic, but it should give some feeling of whether it is common for the system to reach symmetric points. Again, it provides a model, relatively simple to analyze, in which one can assess whether there is or is not a net drift towards the symmetric points. We have seen that plausibly an order one fraction of states lie within the neighborhood of symmetric points. However, we have also seen that for non-R symmetries, this fact is of no particular importance. Even if the system starts in the neighborhood, the system will drift towards non-symmetric, AdS states.

For $\mathrm{R}$ symmetric states, the situation is potentially quite different. We have investigated, first, the question of whether there are likely to be predominantly dS or AdS states in the neighborhood. Within the models we have studied, both situations arise, so it seems plausible that, for a finite fraction of $\mathrm{R}$ symmetric points, the neighborhood is populated by metastable dS states. In this case, we have seen, that typical states in the neighborhood will drift towards a symmetric state. We take this to mean that an order one fraction of possible starting points leave the system in a symmetric state. Thus, even though such states are rare, cosmological considerations are likely to favor them.

Based on this study, we offer the following tentative conclusions:

1. An underlying landscape is likely to lead to low energy supersymmetry, simply because supersymmetry seems the most generic way to guarantee stability.

2. Discrete $\mathrm{R}$ symmetries might plausibly emerge in a landscape. Even though these states are far less numerous than states without such symmetries, they are likely to be cosmological attractors in a finite fraction of cases. As discussed in [19], they should tend to exhibit supersymmetry breaking at low energies, and there can easily be enough of them to resolve the cosmological constant problem.

3. Discrete non-R symmetries (as one might want to understand, say, the structure of the NMSSM or some flavor structure) do not seem favored in any particular way. They are 
rare, and don't seem to be singled out by (at least naive) cosmological considerations.

4. Slightly increasing the size of the discrete symmetry does not seem to lead to a significant suppression, so intricate discrete symmetries (such as might suppress dimension five proton decay) might plausibly arise.

As we have explained, establishing these statements as an ironclad "top down" prediction is difficult, but it seems likely that further landscape studies could well establish that they are robust.

In this paper, we have focussed principally on discrete symmetries. The problem of gauge symmetries is of obvious interest. In field theory, it is well-known that finite temperature tends to favor the maximal unbroken gauge symmetry. The issues, in the landscape context, are different and somewhat more complicated than those connected with discrete symmetry, and require a separate investigation, which will be reported elsewhere. 


\section{Acknowledgements}

We acknowledge valuable conversations with Tom Banks, Ben Freivogel, and Eva Silverstein. We thank Matt Johnson for sharing with us some of his concerns about IIB theories and eternal inflation, which bear some similarity to issues raised here, and Leonard Susskind and Steve Shenker for asking us several important, focussed questions. This work supported in part by the U.S. Department of Energy.

\section{References}

[1] S. Weinberg, Rev. Mod. Phys. 61, 1 (1989).

[2] R. Bousso and J. Polchinski, JHEP 0006, 006 (2000) [arXiv:hep-th/0004134].

[3] S. Kachru, R. Kallosh, A. Linde and S. P. Trivedi, Phys. Rev. D 68, 046005 (2003) [arXiv:hep-th/0301240].

[4] L. Susskind, arXiv:hep-th/0302219.

[5] L. Susskind, The Cosmic Landscape, Little Brown, New York, 2005.

[6] K. Becker and M. Becker, Nucl. Phys. B 477, 155 (1996) [arXiv:hep-th/9605053]; J. de Boer, R. Dijkgraaf, K. Hori, A. Keurentjes, J. Morgan, D. R. Morrison and S. Sethi, Adv. Theor. Math. Phys. 4, 995 (2002) [arXiv:hep-th/0103170]; S. B. Giddings, S. Kachru and J. Polchinski, Phys. Rev. D 66, 106006 (2002) [arXiv:hep-th/0105097]; B. S. Acharya, arXiv:hep-th/0212294 and arXiv:hep-th/0303234.

[7] O. DeWolfe, A. Giryavets, S. Kachru and W. Taylor, JHEP 0507, 066 (2005) [arXiv:hepth/0505160].

[8] F. Denef and M. R. Douglas, JHEP 0405, 072 (2004) [arXiv:hep-th/0404116].

[9] F. Denef and M. R. Douglas, JHEP 0503, 061 (2005) [arXiv:hep-th/0411183].

[10] M. Dine, D. O’Neil and Z. Sun, JHEP 0507, 014 (2005) [arXiv:hep-th/0501214].

[11] See, e.g. A. Saltman and E. Silverstein, JHEP 0411, 066 (2004) [arXiv:hep-th/0402135].

[12] M. R. Douglas, arXiv:hep-th/0405279.

[13] L. Susskind, arXiv:hep-th/0405189. 
[14] M. Dine and Z. Sun, JHEP 0601, 129 (2006) [arXiv:hep-th/0506246].

[15] M. Dine, G. Festuccia, A. Morisse and K. van den Broek, JHEP 0806, 014 (2008) [arXiv:0712.1397 [hep-th]].

[16] S. Ashok and M. R. Douglas, JHEP 0401, 060 (2004) [arXiv:hep-th/0307049].

[17] T. Clifton, S. Shenker and N. Sivanandam, JHEP 0709, 034 (2007) [arXiv:0706.3201 [hepth]].

[18] L. J. Hall and Y. Nomura, arXiv:0712.2454 [hep-ph].

[19] M. Dine, E. Gorbatov and S. D. Thomas, arXiv:hep-th/0407043.

[20] S. R. Coleman, Phys. Rev. D 15, 2929 (1977) [Erratum-ibid. D 16, 1248 (1977)].

[21] S. R. Coleman and F. De Luccia, Phys. Rev. D 21, 3305 (1980).

[22] S. Kachru, M. B. Schulz and S. Trivedi, JHEP 0310 (2003) 007 [arXiv:hep-th/0201028]. 